\title{
THE ALEXANDER AND MARKOV THEOREMS VIA DIAGRAMS FOR LINKS IN 3-MANIFOLDS
}

\author{
PAUL A. SUNDHEIM
}

\begin{abstract}
Let $M$ be a 3-manifold with an open book decomposition. We obtain a new proof that a link in $M$ has a braided form and that two braided forms are related by a sequence of two Markov moves for $M$ by generalizing Morton's approach for links in $S^{3}$.
\end{abstract}

In 1923 Alexander proved a theorem [1] stating that any oriented link in $S^{3}$ is equivalent to the closure of some braid in the braid group $B_{n}$. Markov later proved in 1935 [7] that any two such braids for equivalent links are related by a sequence of two moves. Both of these results are proven more thoroughly in a book by Birman [3]. Morton [8] discovered a new technique for proving these theorems using diagrams and something which he called a threading of the diagram. In 1983 Rudolph [11] generalized Alexander's theorem on the existence of a braided form of a link to arbitrary 3-manifolds. Skora later generalized both Alexander's, and Markov's theorem using Birman's approach (see Richard Skora [12]). This article gives a new proof of Skora's generalizations using Morton's methods and follows Morton's paper closely.

The technique used in generalizing these theorems has as its base a theorem proven by Alexander [1] which states that any closed orientable 3-manifold contains a link whose complement fibers over $S^{1}$ with the fiber being an orientable surface. In 1978 Myers [9] and F. Gonzalez-Acuna proved that the link can be chosen to be a knot, and this result was extended to the nonorientable case in Berstein and Edmonds' paper [2]. Thus we may project a link in the manifold (after an adjustment) to one of the fibers to obtain a diagram. The process of obtaining a diagram for a link and their equivalence is described in the paper [13]. The definition of $R$-move and the result are given on pages 592 and 593.

The existence of a braided form for a link in a 3-manifold $M$ is proven in Theorem 2 and the description of how two braided forms for a link are related is shown in Theorem 6. This is done by describing an equivalence between braided forms and then describing this equivalence in terms of operations on elements in the graded braid group.

I would like to thank Cameron Gordon for his invaluable help.

Received by the editors February 1, 1990 and, in revised form, July 12, 1990.

1980 Mathematics Subject Classification (1985 Revision). Primary 57M25; Secondary 20F36. lence.

Key words and phrases. 3-manifold, open book decomposition, link, knot, braid, Markov equiva- 
Notation. We will allow the 3-manifold $M$ to be any manifold of the form $M^{3}=F \times I / \sim$ where

1. $I=[-1,1]$,

2. $F$ is a compact surface with $C$ a boundary component,

3. $\sim$ is the equivalence relation

(a) $(x, t) \sim(x, s)$ if $x \in C$,

(b) $(h(x), 1) \sim(x,-1)$ where $h: F \rightarrow F$ is a homeomorphism which is the identity on a regular neighborhood $U$ of the boundary.

Such a manifold is said to have an open book decomposition with binding $C$ and page $F$. Let $q: F \times I \rightarrow M$ be the quotient map and let $\pi: F \times I \rightarrow F$ be projection $(x, t) \mapsto x$. Let

$$
\begin{aligned}
& N=q(U \times I), \\
& F_{t}=q(F \times\{t\}) \text { for }-1<t<1, \\
& \bar{F}=q(F \times\{1\} \cup F \times\{-1\}), \\
& \bar{C}=q(C \times I) .
\end{aligned}
$$

$K$ will denote a link in $M$ i.e. a PL embedding of a finite number of copies of $S^{1}$. Two links in $M$ will be called equivalent if there is an isotopy of $M$ taking one link to the other. Equivalence of oriented links will require the isotopy to preserve the orientation; this is important in the proof of Theorem 6. The following definition and result described in [13] provides a generalization of the classical Reidemeister moves [10] to the link diagrams for links in an arbitrary manifold with an open book decomposition.

Theorem 1. Any two diagrams for a link are related by a sequence of $R$-moves (They are R-equivalent.)

Definition. A pair of diagrams for $K$ are related by the $R$-move $R_{i}$ if they are the same except for a disk where
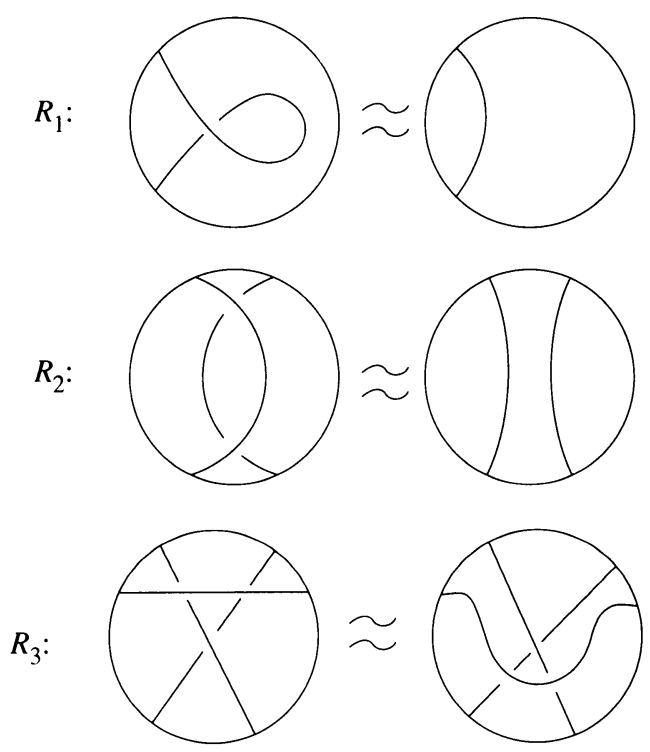

or a portion of the diagram where 

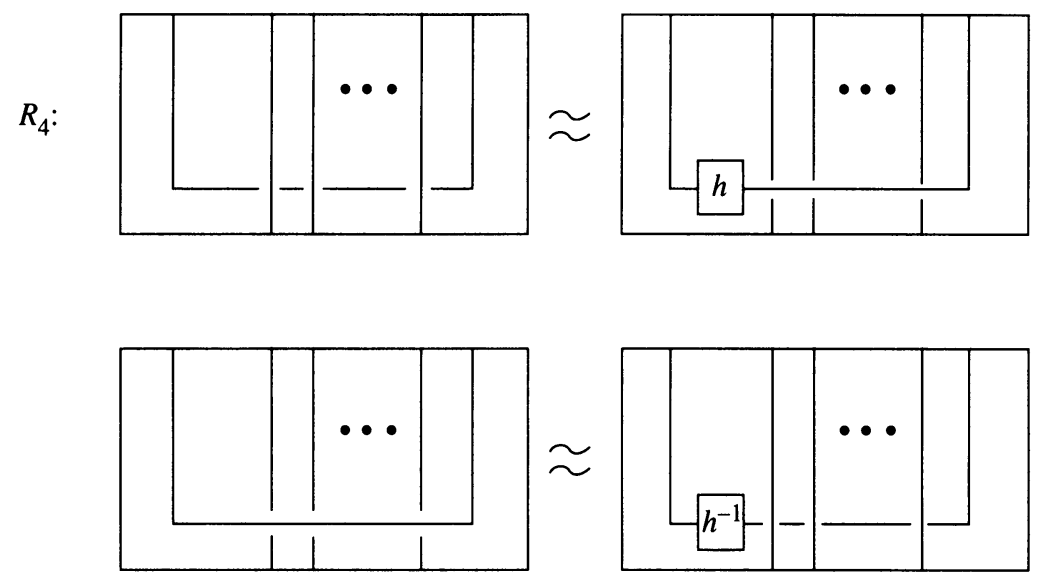

The $h$ indicates the image of the horizontal arc under $h$ and the vertical sides of each rectangle are pieces of $C$.

Definitions. 1. Let $T_{n}=\{1, \ldots, n\}$ and $I=[-1,1]$.

2. A braid on $n$ strands is an embedding of $T_{n} \times I$ in $(F \backslash \partial F) \times I$ that has no local extrema (relative to $I$ ), and where

$$
\pi\left(\operatorname{Im}\left(T_{n} \times\{-1\}\right)\right)=\pi\left(\operatorname{Im}\left(T_{n} \times\{1\}\right)\right) .
$$

Two braids are equivalent if they are isotopic in $F \times I$ leaving $F \times\{ \pm 1\}$ fixed. See Figure 1 . These braids form a group $B_{n}(F)$ under concatenation. A more detailed description of this operation with generators and relations can be found in [3]. For braids $\alpha$ and $\beta$ the operation is

$$
\alpha \cdot \beta(i, s)= \begin{cases}(\pi(\alpha(i, 2 s)), s) & \text { if } 0 \leq s \leq \frac{1}{2} \\ (\pi(\beta(i, 2 s-1)), s) & \text { if } \frac{1}{2} \leq s \leq 1\end{cases}
$$

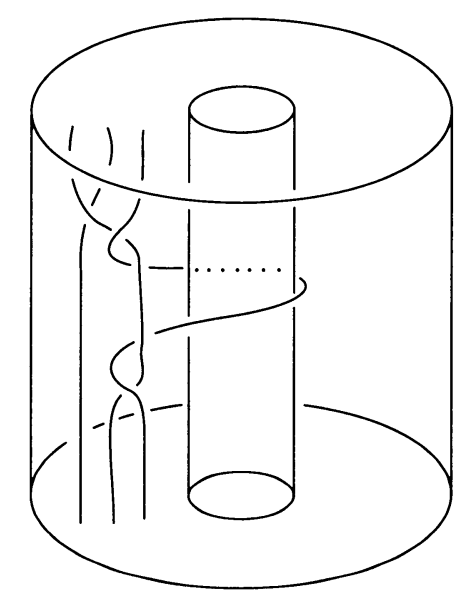

FIGURE 1. An example of a braid where $F$ is an annulus 


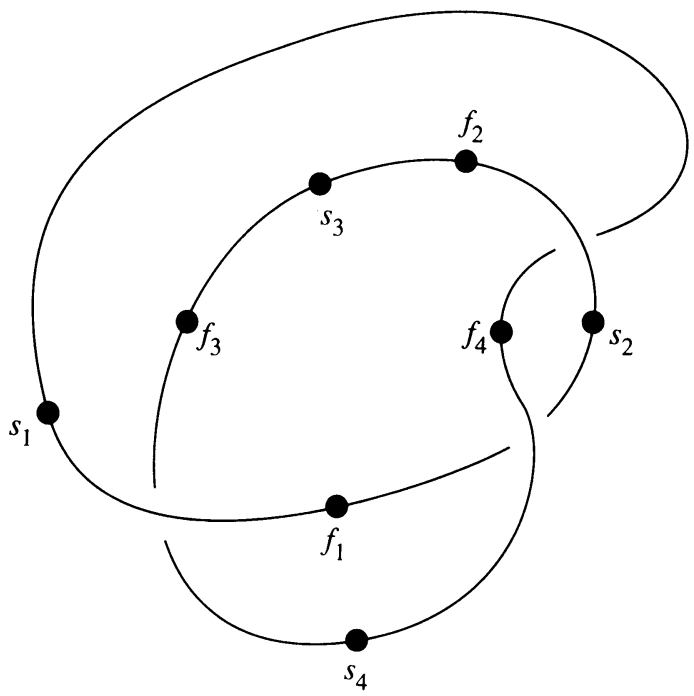

FIGURE 2. An example of an $(\mathscr{S}, \mathscr{F})$ for a trefoil

3. The closure of the braid $\beta$, is $\hat{\beta}$, the image of the braid in $F \times I / \sim=M$. (We many assume that $h$ is the identity on $\beta \cap(F \times\{ \pm 1\})$.)

4. The complete closure of $\beta, \hat{\beta} \cup L$ is $\hat{\beta}$ with a simple closed curve $L$ which is isotopic to $\bar{C}$ in $M \backslash \hat{\beta}$.

Notes. Definition 4 implies that the projection map $\pi_{2}: M \backslash \bar{C} \rightarrow S^{1}$ by $(x, t) \mapsto$ $(\cos (\pi t), \sin (\pi t))$ is locally monotonic on $\hat{\beta}$. Also a technical lemma, Lemma 6.1 , implies that any isotopy giving an equivalence of two closed braids may be assumed to be level preserving. $K$ in $M \backslash \bar{C}$ will be said to be in braided form if $\pi_{2}$ is locally monotonic on $K$.

This defines the process of obtaining a braided form from a braid. We now discuss the process of obtaining a braided form from a diagram. After showing an equivalence between threadings and braided forms (Theorems 2 and 3) we may then use diagrams to relate braided forms.

Definition. 1. An oriented link $K \cup L$ is braided (relative to the simple closed curve $L$ in $F_{0}$ ) if there is an isotopy, $\psi$ of $M$ taking $K \cup L$ to $K^{\prime} \cup \bar{C}, L$ to $\bar{C}$ in $F_{0}$, and such that $\pi_{2} \circ \psi$ is locally increasing on $K^{\prime} . K \cup L$ is then called a braided link.

2. Let $D(K)$ be a diagram for $K$ in $F_{0}$. Orient $D(K)$. A choice of overpasses for $D(K)$ is a pair of subsets $\mathscr{S}=\left\{s_{1}, \ldots, s_{n}\right\}$ and $\mathscr{F}=\left\{f_{1}, \ldots, f_{n}\right\}$ the starting and finishing points of overpasses which alternate in $D(K)$, which divide $D(K)$ into arcs $[s, f]$ called overpasses containing no undercrossings, underpasses $[f, s]$ containing no overcrossings, and so that each component of $D(K)$ has at least one point of $\mathscr{S}$ and $\mathscr{F}$. See Figure 2. Notice that since $D(K) \subseteq \operatorname{Int}\left(F_{0}\right)$ then $(\mathscr{S} \cup \mathscr{F}) \cap \partial F_{0}=\varnothing$.

3. A threading of a diagram $D(K)$ in $F_{0}$ with $(\mathscr{S}, \mathscr{F})$ is a diagram of a link $K \cup L$ where $L$ is an unknotted simple closed curve in $F_{0}$ such that:

(a) $L=\bar{C}$ except for an arc which forms simple closed curves $(L \backslash \bar{C}) \cup(\bar{C} \backslash L)$ bounding disks $A_{1}, \ldots, A_{l}$ in $F$. Let $A$ be $\bigcup_{i=1}^{l} A_{i}$. 


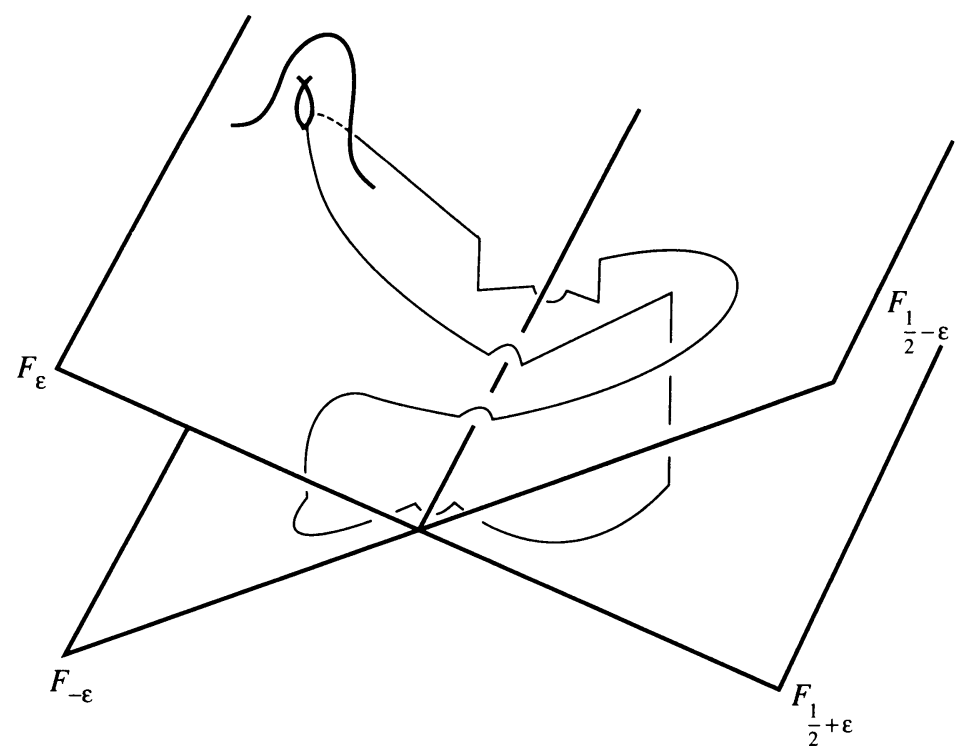

FIGURE 3. A braided link form obtained from a threading of a diagram

(b) $\mathscr{F} \subseteq A, \mathscr{S} \subseteq F_{0} \backslash \operatorname{Int}(A)$.

(c) $\pi\left(q^{-1}(L)\right)$ intersects $\pi\left(q^{-1}(K)\right)$ transversely.

(d) $D(K)$ crosses over $L$ from $\mathscr{S}$ to $\mathscr{F}, D(K)$ crosses under $L$ from $\mathscr{F}$ to $\mathscr{S}$.

Theorem 2. A threading of a diagram represents a braided link.

Proof. Let $K \cup L$ be a link that is represented by a threading in $F_{0}$ with $(\mathscr{S}, \mathscr{F})$. Using the definition of a threading define an isotopy $\psi$ of $M$ taking $L$ to $\bar{C}$ in $F_{0}$ across $A$; the union of the disjoint disks bounded by $(\bar{C} \backslash L) \cup$ $(L \backslash \bar{C})$. This isotopy can be further required to take $\mathscr{S}$ into $F_{1 / 2}$ across $A$.

Project $\left[s_{i}, f_{i}\right]$ to $F_{\varepsilon} \cup F_{1 / 2-\varepsilon}$.

Project $\left[f_{i}, s_{i}\right]$ to $F_{-\varepsilon} \cup F_{1 / 2+\varepsilon}$.

Connect $s_{i}$ in $F_{1 / 2+\varepsilon}$ to $s_{i}$ in $F_{1 / 2-\varepsilon}$ by $q\left(\left\{s_{i}\right\} \times[1 / 2-\varepsilon, 1 / 2+\varepsilon]\right)$. Use $q\left(\left\{f_{i}\right\} \times[-\varepsilon, \varepsilon]\right)$ for $\mathscr{F}$. Move $\left[s_{i}, f_{i}\right]$ over $L$ near $L$ and $\left[f_{i}, s_{i}\right]$ under $L$ near $L$. See Figure 3. Now $\pi_{2}$ is locally monotone on $K$ so that $K \cup L$ is braided.

Note. We may now take any link, produce a diagram, construct a threading, obtain a braided link using Theorem 2 and then get a braid by surguring along $\bar{F}$. Thus Alexander's theorem stated on the first page of this paper is proved in the manifold $M$. It now remains to show that any braided form for a link can be expressed in terms of a threading (Theorem 3) and then to see how two braids for a link obtained by threadings are related (Theorem 6 ). This is done by relating braided links in $M$ by a sequence of two moves (defined later) and then analyzing the two moves as moves in the braid group.

Theorem 3. Given a link $K$ which is equivalent to $\hat{\beta}$ where $\beta \in B_{n}(F)$ there is a threading of a diagram for $K$ which corresponds to $\beta$. 


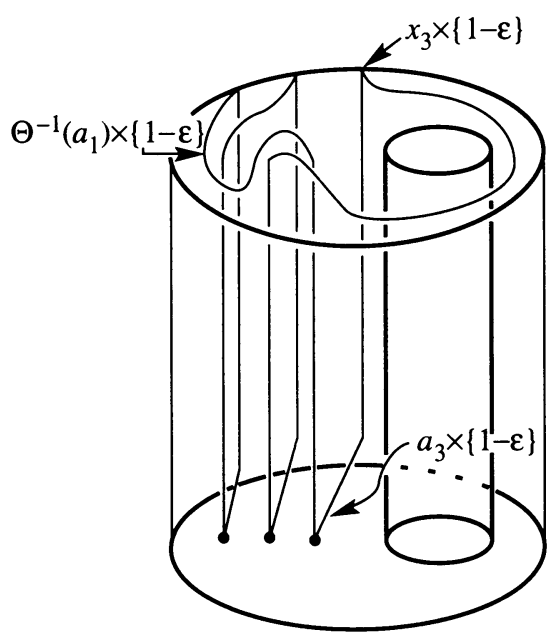

Figure 4. An example of a $K^{\prime}$ where $F$ is an annulus

Proof. Recall that $\pi: F \times I \rightarrow F$ is given by $\pi(x, t)=x$. Let $Q_{n}=\left\{q_{1}, \ldots, q_{n}\right\}$ $=\pi(\beta \cap(F \times\{1\}))$. More explicitly let $\gamma:[0,1] \rightarrow F$ be an arc so that

$$
\gamma(0) \in \partial F, \quad \gamma(1)=q_{1}, \quad \gamma\left(\frac{1}{2}\right)=q_{2}, \ldots, \gamma\left(\frac{1}{n}\right)=q_{n} .
$$

$\beta$ in $F \times I$ corresponds to an isotopy $\theta$ of $Q_{n}$ in $F$ so that $\theta\left(Q_{n}, t\right)=$ $\pi(\beta \cap(F, t))$. By the isotopy extension theorem $\theta$ can be extended to an isotopy given by $\theta: F \times I \rightarrow F$. $\theta$ corresponds to a level preserving homeomorphism Q: $F \times I \rightarrow F \times I$ given by $\boldsymbol{\theta}(x, t)=(\theta(x, t), t)$. We may assume that $\boldsymbol{\theta}$ is given by the identity on $(\partial F \times I) \cup(F \times\{-1\})$.

Let $\boldsymbol{\Theta}_{1}: F \rightarrow F$ be given by $\pi(\Theta(x, 1))$. Let $A$ be a disk in $F$ containing $\gamma$ whose boundary shares an arc with $C$. Let $a_{1}, \ldots, a_{n}$ be disjoint arcs from $q_{1}, \ldots, q_{n}$ in $A$ to $C$. Let $x_{1}, \ldots, x_{n}$ be the endpoints of $a_{1}, \ldots a_{n}$ on $C$. Let $a=\bigcup_{i=1}^{n} a_{i}$.

For some small $\varepsilon>0$ let $K^{\prime}$ in $F \times I$ be the link

$$
\begin{aligned}
K^{\prime}= & \Theta_{1}^{-1}(a \times\{1-\varepsilon\}) \cup(a \times\{-1+\varepsilon\}) \\
& \cup\left(Q_{n} \times[-1+\varepsilon, 1-\varepsilon]\right) \cup\left(\left\{x_{1}, \ldots, x_{n}\right\} \times[-1+\varepsilon, 1-\varepsilon]\right) .
\end{aligned}
$$

See Figure 4. Let $\overline{K^{\prime}}$ be the image of $K^{\prime}$ under $q: F \times I \rightarrow M$.

Claim: $\overline{K^{\prime}}$ is equivalent to $\hat{\beta}$. Apply $\Theta$ to $K^{\prime}$. The image in $F \times\{1-\varepsilon\}$ is $a \times\{1-\varepsilon\}$ and the image in $F \times\{-1+\varepsilon\}$ is $a \times\{-1+\varepsilon\} . \Theta$ is the identity on $\partial F \times I$ so $\left\{x_{1}, \ldots, x_{n}\right\}$ remains unchanged and $Q_{n} \times[-1+\varepsilon, 1-\varepsilon]$ becomes $\beta$ in $F \times[-1+\varepsilon, 1-\varepsilon]$.

Since $a \subseteq A$ and $h$ may be assumed to be the identity on $A$ then $\overline{K^{\prime}}=q\left(K^{\prime}\right)$ is equivalent to $\hat{\beta}$ by isotopy across the disjoint disks

$$
q\left(a_{i} \times(I \backslash(-1+\varepsilon, 1-\varepsilon))\right)
$$

as was claimed.

Move the points of $\overline{K^{\prime}}$ slightly off $\bar{C}$ in $F_{0} \cap N$. A diagram for $K^{\prime}$ then is just a regular projection $M \backslash \bar{F} \rightarrow F$ of $\overline{K^{\prime}} \subseteq q(\operatorname{Int}(F) \times[-1+\varepsilon, 1-\varepsilon])$ into $F$ recording the crossings of $\overline{K^{\prime}}$. Since $K$ is equivalent to $K^{\prime}$ then this diagram is a diagram of $K$. Call it $D(K)$. 


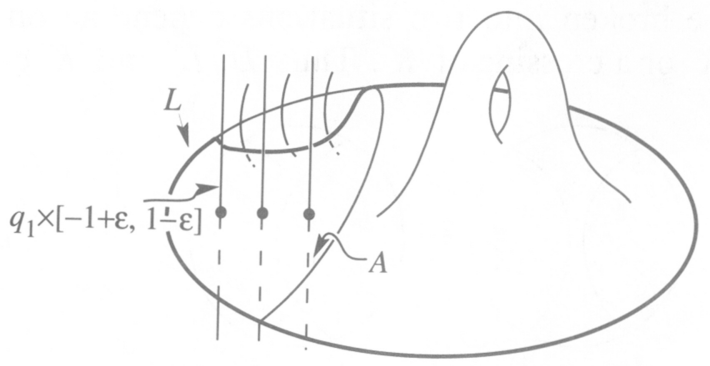

Figure 5. An example of $K^{\prime} \cup L$ near $F_{0}$

Let $L=\bar{C}$ except in $q(A \times\{0\})$ where it runs near $\bar{C}$ in $N \cap F_{0}$. See Figure 5. Then $\overline{K^{\prime}} \cup L$ is a braided link relative to $L$. Let $(\mathscr{S}, \mathscr{F})=$ $\left(Q_{n},\left\{x_{1}, \ldots, x_{n}\right\}\right)$. After $(\mathscr{S}, \mathscr{F})$ together with $L$ are moved slightly off $C$ in $F \cap N$ in the same manner as $\overline{K^{\prime}}$ then $L$ is a threading of $D(K)$.

Definition. Let $K \cup L$ and $K^{\prime} \cup L^{\prime}$ be braided links. $K \cup L$ and $K^{\prime} \cup L^{\prime}$ are Markov equivalent if they are related by a sequence of the following moves:

1. Isotopy in $M$.

2. $K \cup L$ and $K^{\prime} \cup L^{\prime}$ are identical except for arcs $\alpha \subset K$ and $\alpha^{\prime} \subseteq K^{\prime}$ where

(a) $p=\pi_{2} \circ \psi$ (where $\psi$ is as in the definition of a braided link) has

(i) $p(\alpha)=$ pt. ,

(ii) $p \mid(K \backslash \alpha)$ is locally monotonic,

(iii) $p$ is monotonic of degree 1 on $\alpha^{\prime}$ and

(b) $\exists$ disk $A$ such that:

(i) $\partial A=\alpha \cup \alpha^{\prime}$,

(ii) $($ Int $A) 历 L=$ pt. ,

(iii) $($ Int $A) \cap\left(K \cup K^{\prime}\right)=\varnothing$.

This move will be called stabilization.

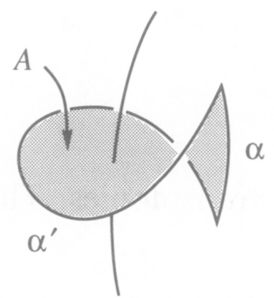

Note. Now we prove that any two braided link forms for a link are Markov equivalent. The method of proof is first to fix a diagram and allow the threading to vary:

Theorem 4. Any two threadings of a given diagram for $K$ are Markov equivalent. and secondly to vary the diagrams and show there are equivalent threadings (Theorem 5). Theorem 4 is proven in two steps:

1. Fixing a set of overpasses (Lemma 4.1).

2. Given two sets of overpasses there is a third relative to which any threading is a threading of the first two (Lemma 4.2).

Lemma 4.1. Given a diagram $D(K)$ of an oriented link $K$ with choice of overpasses $(\mathscr{S}, \mathscr{F})$ the threadings defined by any two simple closed curves $L$ and $L^{\prime}$ are Markov equivalent.

Proof. Let $A$ be the union of the disks bounded by $(C \backslash L) \cup(L \backslash C)$ and $A^{\prime}$ be the union of disks bounded by $\left(C \backslash L^{\prime}\right) \cup\left(L^{\prime} \backslash C\right)$. Then $\mathscr{F}$ lies in $A \cap A^{\prime}$.

Case a: Suppose that $L$ and $L^{\prime}$ are isotopic in $F \backslash(\mathscr{S} \cup \mathscr{F})$. An isotopy taking the simple closed curve $L$ to the simple closed curve $L^{\prime}$ across the 
diagram for $K$ can be broken into two situations depending on whether $L$ encounters an arc of $K$ or a crossing of $K$. Thus $L, L^{\prime}$ and $K$ are related by a sequence of moves

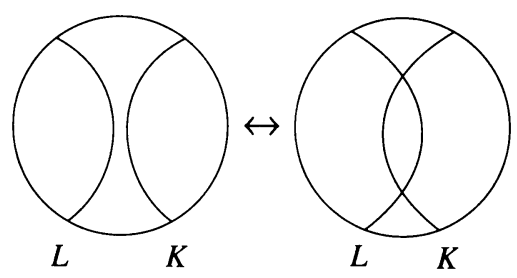

type 1

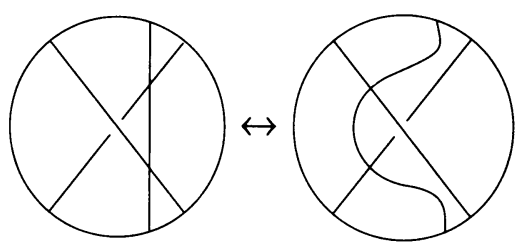

type 2

Each of the above diagrams represent four situations depending on whether an intersection of $L$ with $K$ is chosen to cross in each of the two ways.

The type 1 moves

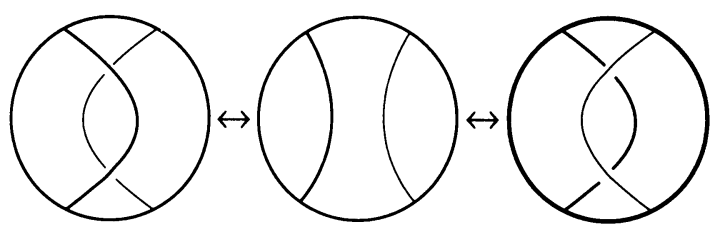

are isotopies. The type 1 moves

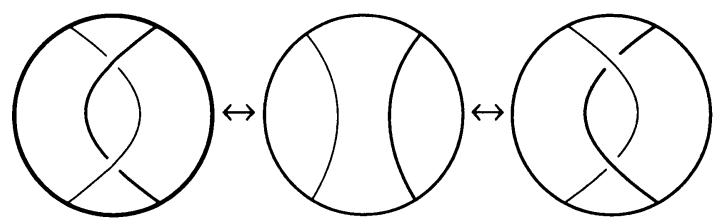

are stabilizations. The type 2 moves

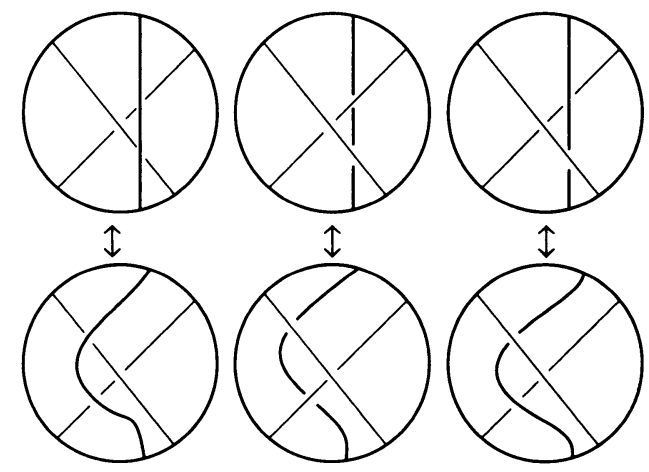

are isotopies. Finally, 


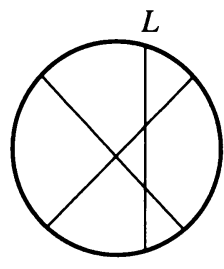

stabilization

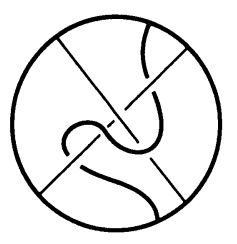

isotopy
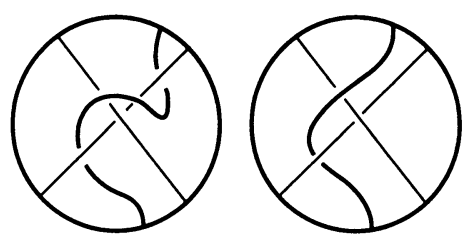

stabilization

Case b: General case. Suppose $L_{i} \rightarrow L_{i+1}$ is an intermediate step in $L \rightarrow L^{\prime}$ obtained by pushing a pair of adjacent crossings with $K$ past a point of $\mathscr{F}$ (or $\mathscr{S}$ ) as in Figure 6. Then $L_{i}$ and $L_{i+1}$ define isotopic threadings.

Draw disjoint arcs $a_{1}, \ldots, a_{n}$ in $A^{\prime}$ from the points of $\mathscr{F}$ to the arc $C \backslash L^{\prime}$. Since $\mathscr{F}$ lies in $A \cap A^{\prime}$ then each $a_{i}$ intersects $L^{\prime}$ an even number of times. By a slight isotopy of $F$ we may assume that $K$ lies along $a_{i}$ near $f_{i}$.

Move $L^{\prime}$ so that the points of intersection of $\left\{a_{i}\right\}$ with $L^{\prime}$ are in $K \cap$ $\left(\bigcup_{i=1}^{n} a_{i}\right)$. This may be done in the complement of $\mathscr{S} \cup \mathscr{F}$. A stabilization move may be required, for the points of $a_{i} \cap L^{\prime}$. See Figure 7. Move the intersections across the points of $\mathscr{F}$ a pair at a time until $\left(\bigcup_{i=1}^{n} a_{i}\right) \cap L^{\prime}=\varnothing$. Since $\bigcup_{i=1}^{n} a_{i}$ now lies in both $A$ and the new $A^{\prime}$ then this new $L^{\prime}$ is a curve that is isotopic to $L$ in $F \backslash(\mathscr{S} \cup \mathscr{F})$.

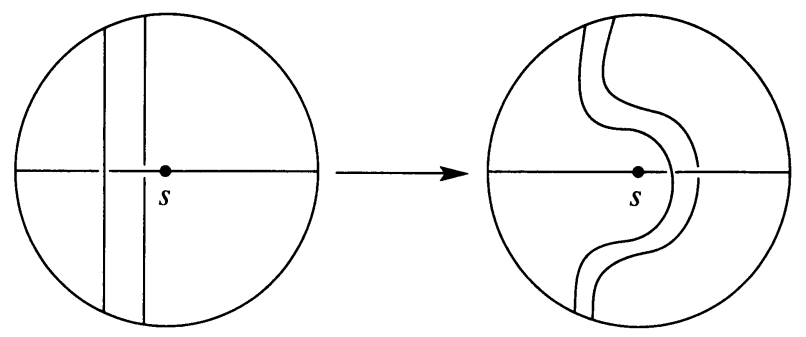

FIGURE 6. Moving $L$ to be isotopic to $L^{\prime}$ in $F \backslash(\mathscr{S} \cup \mathscr{F})$

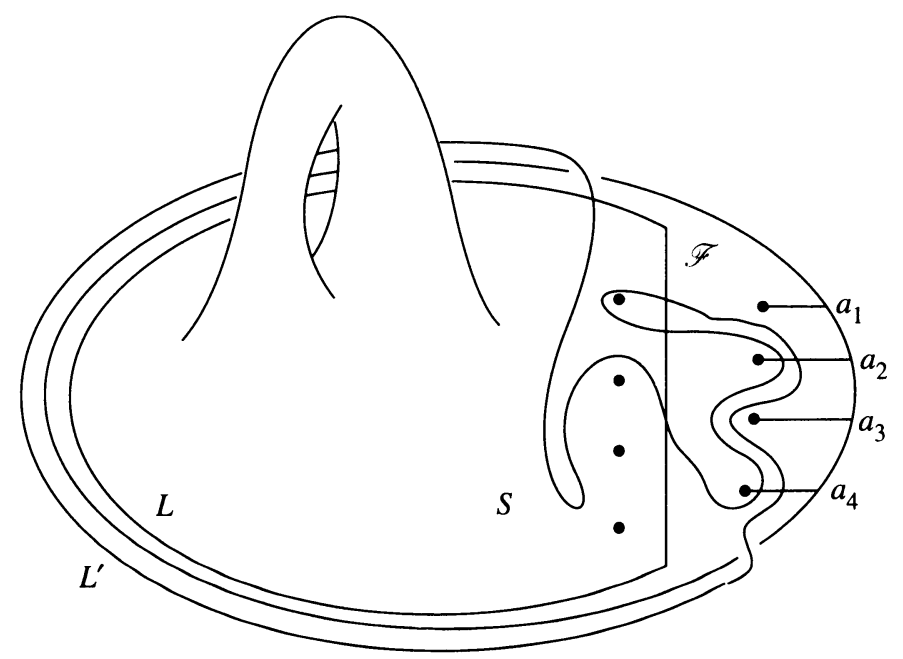

FIGURE 7. Moving one threading for a diagram to another 
Lemma 4.2. Given a diagram for an oriented link $K$ in $F$ and two choices of overpasses $(\mathscr{S}, \mathscr{F})$ and $\left(\mathscr{S}^{\prime}, \mathscr{F}^{\prime}\right)$ there is a simple closed curve that is a threading for both.

Proof. Suppose $(\overline{\mathscr{S}}, \overline{\mathscr{F}})$ is a choice of overpasses and $s \notin \overline{\mathscr{F}}$ is a point of $D(K)$ then we can make a new choice of overpasses $(\overline{\overline{\mathscr{S}}}, \overline{\overline{\mathscr{F}}})$ with $s \in \overline{\overline{\mathscr{S}}}$, $\overline{\mathscr{F}} \subseteq \overline{\bar{F}}$ and $\overline{\mathscr{S}} \subseteq \overline{\overline{\mathscr{S}}}$ by

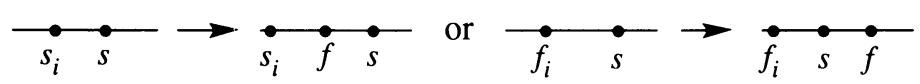

Do this for each point of $\left(\mathscr{S} \backslash \mathscr{S}^{\prime}\right) \cup\left(\mathscr{S}^{\prime} \backslash \mathscr{S}\right)$ and $\left(\mathscr{F} \backslash \mathscr{F}^{\prime}\right) \cup\left(\mathscr{F}^{\prime} \backslash \mathscr{F}\right)$ to get a choice of overpasses $\left(\mathscr{S}^{\prime \prime}, \mathscr{F}^{\prime \prime}\right)$ with $\mathscr{S}^{\prime} \cup \mathscr{S}^{\prime} \subseteq \mathscr{S}^{\prime \prime}$ and $\mathscr{F} \cup \mathscr{F}^{\prime} \subseteq \mathscr{F}{ }^{\prime \prime}$.

Suppose that $L^{\prime \prime}$ is a threading for $\left(\mathscr{S}^{\prime \prime}, \mathscr{F}^{\prime \prime}\right)$. Clearly $L^{\prime \prime}$ separates $\mathscr{S}$ from $\mathscr{F}$ and $\mathscr{S}^{\prime}$ from $\mathscr{F}^{\prime}$. Since $D(K)$ passes over $L^{\prime \prime}$ when going from the side of $L^{\prime \prime}$ containing $\mathscr{S}^{\prime \prime}$ to $\mathscr{F}$ " and under from $\mathscr{F}$ " to $\mathscr{S}^{\prime \prime}$ it does so for $\mathscr{S} \cup \mathscr{S}^{\prime}$ and $\mathscr{F} \cup \mathscr{F}^{\prime}$.

Thus $L^{\prime \prime}$ is a threading for both $(\mathscr{S}, \mathscr{F})$ and $\left(\mathscr{S}^{\prime}, \mathscr{F}^{\prime}\right)$.

This proves Lemma 4.2 and with Lemma 4.1 it proves Theorem 4.

Theorem 5. Any two diagrams for $K$ have Markov equivalent threadings.

Proof. It suffices to show that any two diagrams related by an $R$-move have Markov equivalent threadings.

By Theorem 4 we may make any convenient choice of overpasses.

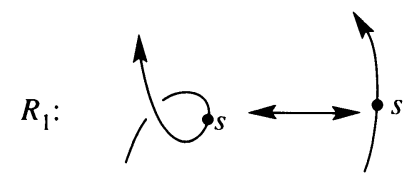

By choosing $\mathscr{S}$ or $\mathscr{F}$ as above and identical elsewhere in the diagram we see that the threadings are the same.

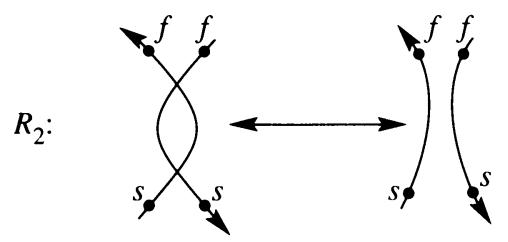

Choose $\mathscr{S}$ and $\mathscr{F}$ as above.

The following threadings are isotopic:

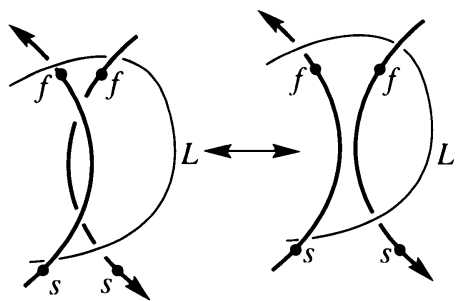

The other cases are similar. 


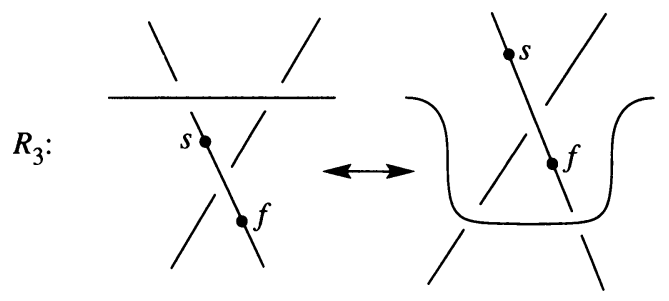

choose $\mathscr{S}$ and $\mathscr{F}$ as above. Choose $L$ as

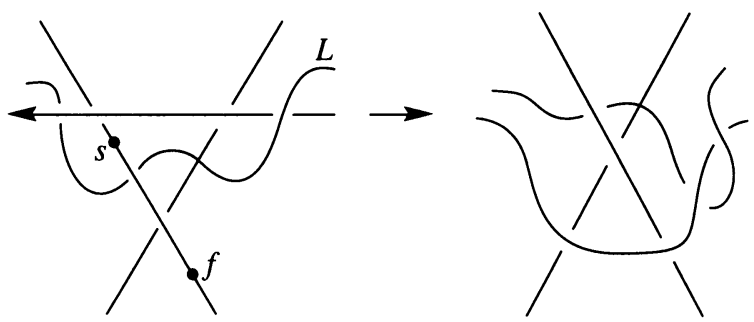

then the threadings are isotopic. The other orientations of the arcs of $D(K)$ as well as the kind of crossing of $L$ with the bottom part of $K$ are irrelevant. The other cases are similar. Finally,

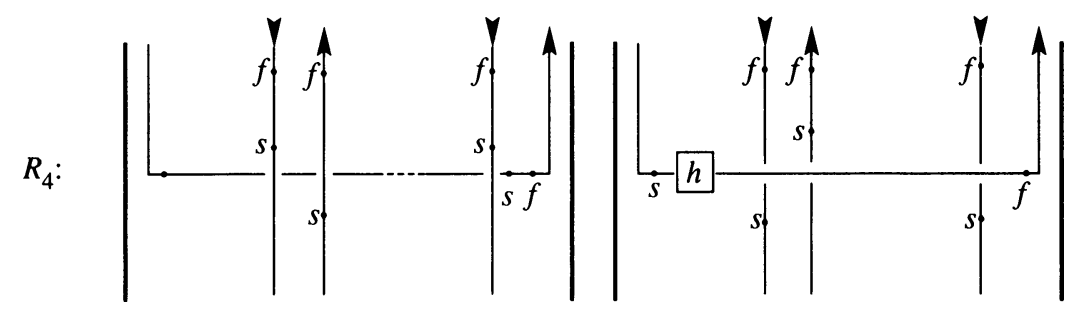

Choose the overpasses as above.
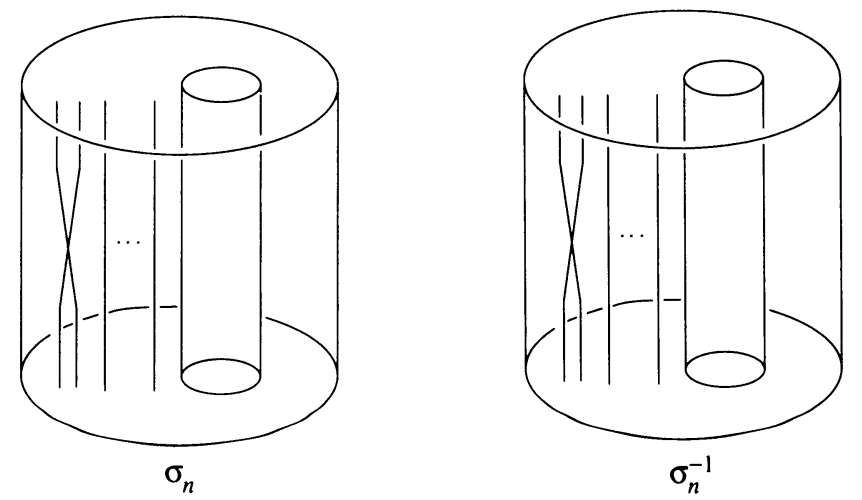

FIGURE 8. $\sigma_{n}$ and $\sigma_{n}^{-1}$ in the braid group for an annulus on $n+1$ strands 
Let $L$ be

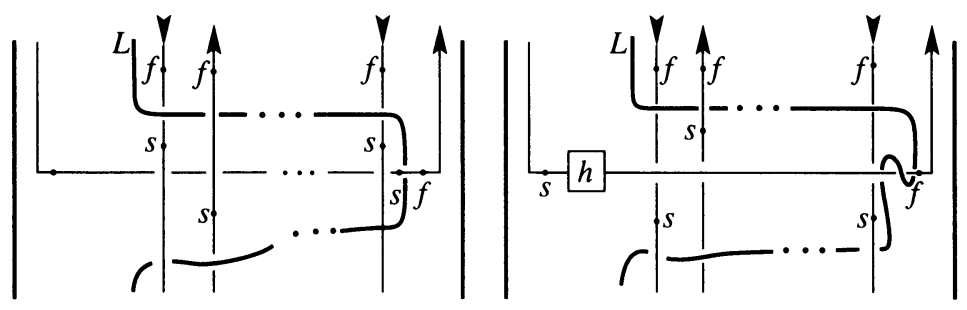

As in the other cases the threading is carried along with the isotopy defining the $R$-move in $M$. Since $L$ and $C$ bound an annulus and the isotopy placing the arc $a$ defined by the $h$ in the $R_{4}$ move is required to place $a$ outside this annulus. Furthermore the over and underpasses of $K$ are either shrunk or stretched to place the points of $\mathscr{S}$ and $\mathscr{F}$ in a position so that the result is a threading. The other cases of orientations of the arcs of $K$ or the inverse of the $R_{4}$ move are similar.

Note. It now remains to describe Markov equivalence in terms of operations on the braid groups

$$
B_{1}(F) \subseteq B_{2}(F) \subseteq \cdots .
$$

The first definition below describes a certain pair of generators of $B_{n}(F)$ for any $F$.

Definitions. 1. Let $\sigma_{n}$ and $\sigma_{n}^{-1}$ be braids as depicted in Figure 8. Notice that $\pi\left(\sigma_{n}^{ \pm 1}\right)$ is $Q_{n}$ with a circle containing $q_{n}$ and $q_{n+1}$.

2. A Markov move for a braid $\beta \in \beta_{n}(F)$ is either of

(a) $\beta \rightarrow \beta \sigma_{n}^{ \pm}$or $\beta \rightarrow \beta^{\prime}$ where $\beta=\beta^{\prime} \sigma_{n-1}^{ \pm 1}$ and $\beta^{\prime} \in B_{n-1}(F)$.

(b) $\beta \rightarrow \gamma \beta h\left(\gamma^{-1}\right)$ where $h\left(\gamma^{-1}\right)$ is the braid which at the level $F_{t}$ is $h\left(\pi\left(\gamma^{-1} \cap F_{t}\right)\right) \times\{t\}$.

Remarks. Two braids are Markov equivalent if they are related by a sequence of the above moves, called Markov moves for braids. It remains to show that Markov equivalent braided links give Markov equivalent braids, i.e. the isotopy and stabilization moves on a braided link correspond to moves of type one and type two above.

Since $h$ induces an automorphism on $B_{n}(F)$, this second move suggests a question about an equivalence relation on a braid group: let $\phi$ be an automorphism of $B_{n}(F)$. Given $\alpha$ and $\beta$ in $B_{n}(F)$ is there a $\gamma \in B_{n}(F)$ such that $\alpha=\gamma \beta \phi\left(\gamma^{-1}\right)$. For $\phi=\mathrm{id}$ and $F=D^{2}$, this question has been answered [5].

Theorem 6. Any two braids for equivalent oriented links are related by a sequence of Markov moves.

In order to prove this main theorem we need a technical lemma which is a trivial consequence of Morse theory involving the cancellation of critical points:

Lemma 6.1. Given two closed braids $\hat{\beta}_{0}$ and $\hat{\beta}_{1}$ isotopic in $M \backslash \bar{C}$, they are level preserving (relative to the fibration) isotopic in $M \backslash \bar{C}$.

Sketch of proof. Let $F^{\prime}=F \backslash C$ and $M^{\prime}=q\left(F^{\prime} \times I\right)$. Let $\phi:\left(Q_{n} \times S^{1}\right) \times I \rightarrow M^{\prime}$ be the isotopy. Adjust the composition of $\phi$ with the fiber map $M^{\prime} \rightarrow S^{1}$ to be generic in the sense of Cerf. This gives a graphic $G$ in $S^{1} \times I$ (see [4]). 


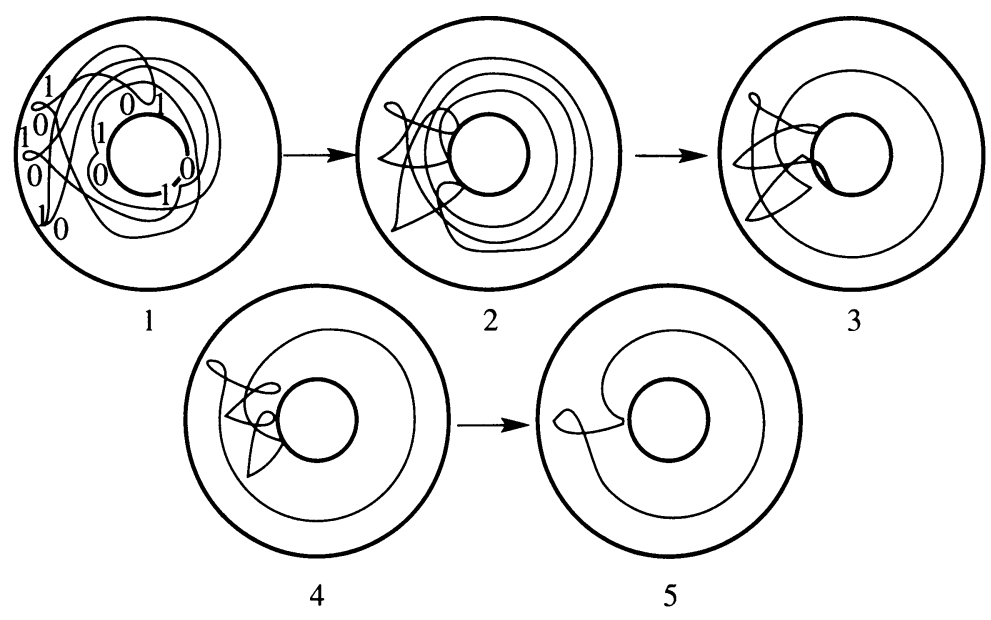

FIGURE 9. An example of reducing a component to having only one birth and death

Arbitrarily assign over and under crossings to the crossings of the graphic. By a component of the graphic we mean a component of the resulting link.

Let $G_{1}$ be a component and suppose it is essential in $S^{1} \times I$. Use the three Cerf lemmas (in much the same way one uses the three Reidemeister moves) to eliminate all but one birth and death while leaving the rest of $G$ fixed. See Figure 9. This is done as follows:

Diagram 1. Move the births of $G_{1}$ near $S^{1} \times\{0\}$ and the deaths near $S^{1} \times\{1\}$.

Diagram 2. By moving the deaths along the paths of the index 0 critical points, level the paths so that they are along some $\{\theta\} \times I$.

Diagram 3. Move the index 0 critical points along the paths of the index 1 critical points so that only one of the index one critical point paths winds around the annulus. This is possible since $G_{1}$ is a closed curve.

Diagram 4. Move all but the topmost births past the essential index 1 critical point path.

Diagram 5. Apply Cerf's dovetail lemma and independence of trajectories for each pair of births and deaths one at a time to cancel all but one birth-death pair.

The last graphic of Figure 9 indicates that the two critical points cancel in some interval $q\left(F^{\prime} \times[s, t]\right)$ keeping $q\left(F^{\prime} \times(I \backslash[s, t])\right)$ fixed. However in the manifold the picture is as in Figure 10. These do not cancel in $q\left(F^{\prime} \times[s, t]\right)$ keeping $q\left(F^{\prime} \times(I \backslash[s, t])\right)$ fixed. Thus $G_{1}$ is not essential and can be removed from $G$.

In this way all of the components of $G$ can be removed and therefore $\phi$ can be assumed to have no local extremes.

Let $F_{\tau}$ be a fiber in $M^{\prime} . F_{\tau} \cap \operatorname{Im}(\phi)$ is a collection of paths which start at points of $\hat{\beta}_{0}$ in $F_{\tau}$ and end at points of $\hat{\beta}_{1}$ in $F_{\tau}$. For $x \in F_{\tau} \cap \hat{\beta}_{0}$ let $\alpha_{x}: I \rightarrow F_{\tau}$ be the corresponding paths. Let $\Phi:\left(\left(Q_{n} \times S^{1}\right) \times I\right) \rightarrow M^{\prime}$ be defined by

$$
\phi((q, \theta), 0)=x \Rightarrow \Phi((q, \theta), t)=\alpha_{x}(t)
$$




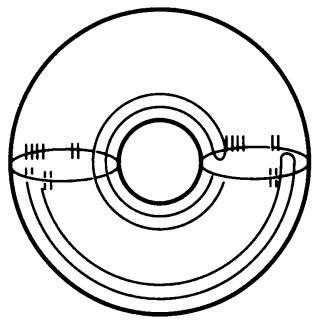

FIGURE 10. A diagrammatic picture of the critical points at their death
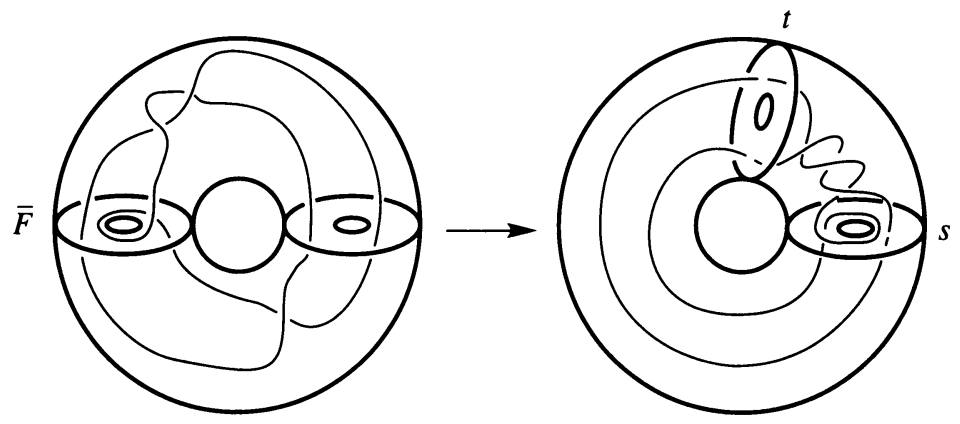

Figure 11. Pushing a closed braid $K$ into the interval $[s, t]$.

Then setwise $\Phi\left(Q_{n} \times S^{1}, t\right)=\phi\left(Q_{n} \times S^{1}, t\right)$ so $\Phi$ is an isotopy taking $\hat{\beta}_{0}$ to $\hat{\beta}_{1}$ and $\Phi$ is level preserving.

Proof of Theorem 6. We must show that any two braided links which are related by isotopy in $M$ or stabilization are related by Markov moves.

Given two equivalent braided links $K \cup L$ and $K^{\prime} \cup L^{\prime}$, both $L$ and $L^{\prime}$ are isotopic to $\bar{C}$ with the isotopy carrying $L$ to $L^{\prime}$ in $F_{0}$. Thus any equivalence taking $K \cup L$ to $K^{\prime} \cup L^{\prime}$ be thought of as an equivalence between two links braided relative to $\bar{C}$. In particular $\psi=\mathrm{id}$.

Case a: Suppose that $\phi$ is an isotopy taking $K \cup \bar{C}$ to $K^{\prime} \cup \bar{C}$ in $M$ keeping $\bar{C}$ fixed. Then $\phi$ restricted to $M \backslash \bar{C}$ takes $K$ to $K^{\prime}$ in $M \backslash \bar{C}$. By the last lemma $\phi$ may be assumed to be level preserving.

Suppose $\bar{F}$ is fixed by $\phi$. This isotopy defines another isotopy in $F \times I$. Since $K$ and $K^{\prime}$ are isotopic in $F \times I$ then they represent the same element in $B_{n}(F)$.

Suppose $q(F \times[s, t]) \nsupseteq \bar{F}$ is fixed by $\phi$. Let $K \cap \bar{F}=Q_{n}=\left\{q_{1}, \ldots, q_{n}\right\}$ and let $\beta$ be a braid for $K$. By an abuse of notation $Q_{n}$ will also be a set in $F$ so that $\pi\left(Q_{n} \times\{ \pm 1\}\right)=Q_{n}$.

Let $\beta_{1}=Q_{n} \times[1,2] \cup \beta \cup Q_{n} \times[-2,1]$ in $F \times[-2,2]$. Take $P: F \times[-2,2] \rightarrow$ $F \times[-1,1]$ to be

$$
P(x, r)= \begin{cases}(x,(r+2)(s+1)-1), & r \leq-1, \\ (x,((r+1)(t-s) / 2)+s), & -1 \leq r \leq 1, \\ (x,(r-1(1-t)+t)), & 1 \leq r\end{cases}
$$




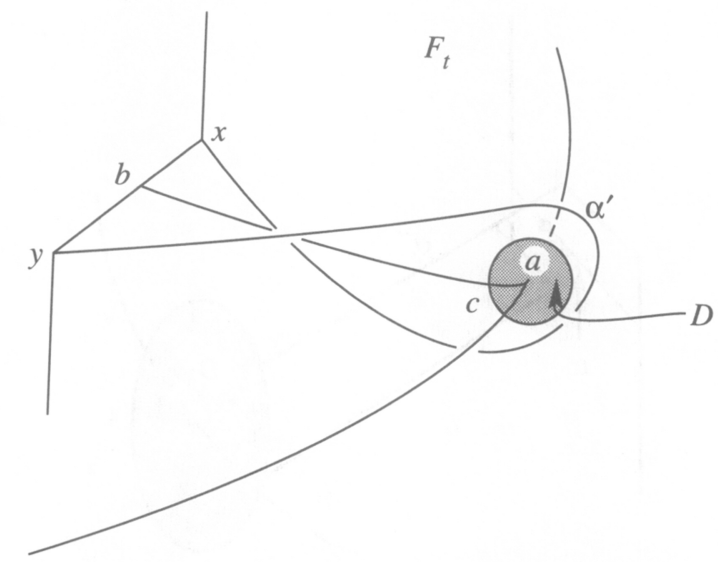

FIGURE 12. Adjusting the stabilization disk $A$

Then $P\left(\beta_{1},[-2,2]\right)$ is just the result of pushing $\beta$ into $F \times[s, t]$. See Figure 11.

Also there is an isotopy $\phi_{1}$ of $M$ which takes $K$ to $K_{1}=q\left(P\left(\beta_{1},[-2,2]\right)\right)$. Notice that $K_{1} \cap q(F \times([-1, s] \cup[t, 1]))=q\left(Q_{n} \times([-1, s] \cup[t, 1])\right)$.

The action on $q\left(Q_{n} \times I\right)$ be $\phi$ is trivial as a braided link so, for some $\gamma \in B_{n}(F), \phi\left(q\left(Q_{n} \times I, 1\right)\right.$ must be $q\left(\gamma \iota h\left(\gamma^{-1}\right)\right)$ going from the levels $[-1, s]$ to $[s, t]$ to $[t, 1]$ respectively where $l$ is the identity $Q_{n} \times[s, t]$. Thus applying $\phi_{1}$ and then $\phi$ to $K$ results in a braid $\gamma \beta h\left(\gamma^{-1}\right)$.

$\phi\left(\phi_{1}(K, 1), 1\right)$ is clearly isotopic to $K^{\prime}=\phi(K, 1)$ since $\phi_{1}(K, 1)=K_{1}$. If $K^{\prime}$ corresponds to the braid $\beta^{\prime}$ then since $\phi_{1}$ fixes $\bar{F}, \beta^{\prime}=\gamma \beta h\left(\gamma^{-1}\right)$.

Any isotopy taking $K$ to $K^{\prime}$ in $M \backslash \bar{C}$ can be broken into isotopies which alternately fix $\bar{F}$ and $q(F \times[s, t])$.

Case b: Suppose that $K \cup \bar{C}$ and $K^{\prime} \cup \bar{C}$ are related by stabilization. Let $\alpha, \alpha^{\prime}$ and $A$ be as in the definition of stabilization.

Since $\pi_{2}$ is constant on $\alpha$ it lies in a particular $F_{t}$. As above we may assume (by applying the isotopy $\phi_{1}$ to $\left.K\right)$ that $K \cap q(F \times[t,-1])=q\left(Q_{n} \times[t,-1]\right)$.

Since $C \cap A=a$ a single point then there is an arc $\gamma$ in $A \cap F_{t}$ which travels from $a$ to a point $b$ of $\alpha$.

Going in the direction of $K$ let $\alpha(0)=x, \alpha(1)=y$ and $\alpha(s)=b$. See Figure 12.

Let $D$ be a small disk in $A$ with center $a$ which we can assume (after an isotopy of $A$ ) to lie as a product of a subarc $\gamma^{\prime} \subseteq \gamma$ with $S^{1}$ in a regular neighborhood of $C$ in $M$. Let the other endpoint of $\gamma^{\prime}\left(\right.$ i.e. $\left.\gamma^{\prime}(0)\right)$ be called $c$. Say $c=\gamma(1-\varepsilon)$.

Define a new arc $\alpha^{\prime \prime}$ as follows (after possibly readjusting $\varepsilon$ ):

$$
\begin{aligned}
\alpha^{\prime \prime}=q\left(\pi\left(q^{-1}(\{x\})\right) \times[t-\varepsilon, t] \cup \pi\left(q^{-1}(\alpha([0, s]))\right) \times\{t-\varepsilon\}\right. \\
\cup \pi\left(q^{-1}\left(\gamma \backslash \gamma^{\prime}\right)\right) \times\{t-\varepsilon\} \cup \pi\left(q^{-1}(\{c\})\right) \times(I \backslash[t-\varepsilon, t+\varepsilon]) \\
\cup \pi\left(q^{-1}\left(\gamma \backslash \gamma^{\prime}\right)\right) \times\{t+\varepsilon\} \cup \pi\left(q^{-1}(\alpha([s, 1]))\right) \times\{t+\varepsilon\} \\
\left.\cup \pi\left(q^{-1}(\{y\})\right) \times[t, t+\varepsilon]\right) .
\end{aligned}
$$

Isotop $\alpha^{\prime}$ to $\alpha^{\prime \prime}$ in $M$. Let $\beta$ and $\beta^{\prime}$ be braids for $K$ and $K^{\prime \prime}=(K \backslash \alpha) \cup \alpha^{\prime \prime}$. See Figure 13. 


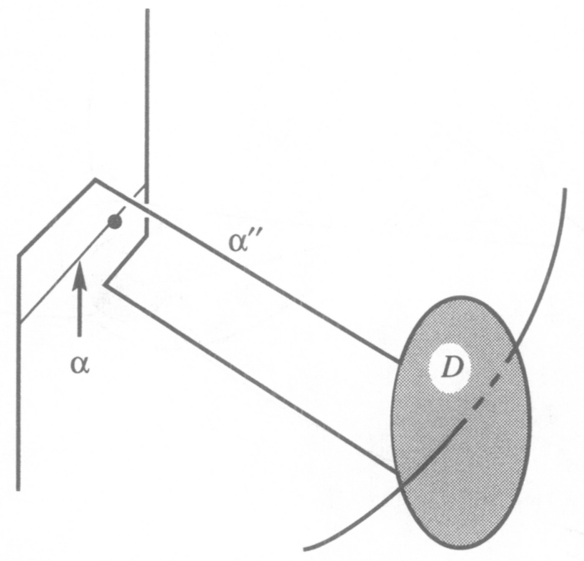

Figure 13. The braids $\beta$ and $\beta^{\prime}$

It remains to show that $\beta^{\prime}$ corresponds to the braid $\beta \sigma_{n}^{ \pm 1}$. Since

$$
\beta^{\prime} \cap F \times[-1, t-\varepsilon]=Q_{n} \times[-1, t-\varepsilon]
$$

we may ignore it and assume that $t=-1+\varepsilon$.

There is a 3-ball about $b$ containing $q\left(\pi\left(q^{-1}(\alpha)\right) \times[-1,-1+2 \varepsilon]\right)$. In $N$ we may adjust $\alpha$ and $\alpha^{\prime \prime}$ so that $b \in F_{t}$ and so that they appear as
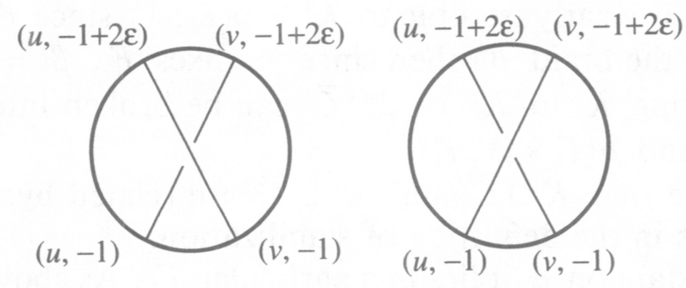

That is $\pi(K \cap N)$ is a simple closed curve with two double points. Isotop $\beta$ in $F \times I$ so that $N$ travels along a regular neighborhood of $\beta$ and so that $b$ moves to $c$. Let $x_{n}=b$. Pick points $\left\{x_{1}, \ldots, x_{n-1}\right\}$ in $\beta \cap F_{t}$ one for each component of $\beta$ in $F_{t}$ not containing $x_{n}$ and let $x_{n+1}=\left(\beta^{\prime} \backslash \beta\right) \cap F_{t}$. Now $\beta^{\prime}: T_{n+1} \times I \rightarrow F \times I$ is such that

1. $\beta(i, t)=\beta^{\prime}(i, t)$ if $(i, t) \in T_{n-1} \times I$,

2. $\beta^{\prime}(n, t)=\beta(n, t)$ if $t \in[-1+2 \varepsilon, 1]$,

3. $\beta^{\prime}(n+1, t)=\left(x_{n+1}, t\right)$ if $t \in[-1+2 \varepsilon, I]$,

4. $\pi\left(\beta^{\prime} \cap N\right)$ is a simple closed curve with

$$
\pi\left(\beta^{\prime}\left(x_{n+1} \times[-1,-1+\varepsilon]\right)\right) \cap \pi\left(\beta^{\prime}\left(x_{n} \times[-1,-1+\varepsilon]\right)\right)=x_{n} \cup x_{n+1} .
$$

This is $\beta \sigma_{n}^{ \pm 1}$.

Remarks. The Markov theorem is $S^{3}$ has been used to prove the invariance of the two-variable. V. Jones polynomial [6]. There is therefore some hope of generalizing this approach to find a more topological proof of Witten's polynomial [14] for links in 3-manifolds using Theorem 6. 


\section{REFERENCES}

1. J. W. Alexander, A lemma on systems of knotted curves, Proc. Acad. Sci. U.S.A. 9 (1923), 93-95.

2. I. Berstein and A. L. Edmonds, On the construction of branched coverings of low-dimensional manifolds, Trans. Amer. Math. Soc. 247 (1979), 87-124.

3. J. Birman, Braids links and mapping class groups, Ann. of Math. Stud., no. 82, Princeton Univ. Press, Princeton, N.J., 1974.

4. J. Cerf, La stratification naturelle des espaces de fonctions differentiables reeles et la theoreme de la pseudo-isotopy, Publ. Math. I.H.E.S. 39 (1970).

5. F. A. Garside, The braid group and other groups, Quart. J. Math. Oxford 20 (1969), 235254.

6. V. F. R. Jones, Hecke algebra representations of braid groups and link polynomials, Ann. of Math. (2) 126 (1987), 335-388.

7. A. A. Markov, Über die freie Aquivalenz der geschlossner Zopfe, Rec. Soc. Math. Moscou 1 (1935), 73-78.

8. H. R. Morton, Threading knot diagrams, Math. Proc. Cambridge Philos. Soc. 99 (1986), 247-260.

9. R. Myres, Open book decompositions of 3-manifolds, Proc. Amer. Math. Soc. 72 (1978).

10. K. Reidemeister, Knotentheorie, Chelsea, New York, 1948.

11. L. Rudolph, Constructions of quasipositive knots and links. I, Monograph. Enseign. Math., 31, Univ. of Genève, 1983.

12. R. K. Skora, Braids in 3-manifolds, (1988).

13. P. A. Sundheim, Reidemeisters theorem for 3-manifolds, Math. Proc. Cambridge Philos. Soc. 110 (1991), 281-292.

14. E. Witten, Quantum field theory and the Jones polynomial, preprint.

Department of Mathematics, University of Wisconsin Center, Marshfield, Wisconsin

E-mail address: psundhei@helios.uwsp.edu 